\title{
Improving Chronic Obstructive Pulmonary Disease (COPD) Symptoms Using a Team-Based Approach
}

\author{
Shaylee Peckens, MD, Megan M. Adelman, PharmD, Amie M. Ashcraft, PhD, MPH, \\ Jun Xiang, MS, Brittany Sheppard, and Dana E. King, MD, MS
}

Background: Chronic obstructive pulmonary disease (COPD) is the third leading cause of death in the United States (US), with West Virginia bearing a disproportionate disease burden. Complex COPD cases can be difficult to manage during a standard primary care provider (PCP) visit, and pharmacological treatment regimens should be individually tailored to each patient.

Methods: To address these needs, the West Virginia University Department of Family Medicine created an interdisciplinary COPD specialty clinic that uses a team-based approach to treat patients with COPD. In order to evaluate the effectiveness of the specialty clinic, we conducted a retrospective chart review to examine the impact of the clinic on patient hospitalizations, emergency department visits, and urgent care visits six months and one year before and after initiating care at the clinic. We also examined the impact of the clinic on patients' self-reported nicotine dependency, COPD symptoms, and tobacco use behavior. Patients referred to the clinic and having at least one visit from February 2015 to February 2019 were included in this study $(n=149)$.

Results: Patients treated at the COPD specialty clinic had significantly fewer hospital admissions and ED visits six months after and one year after initiating care at the clinic as compared to six months before and one year before, respectively. Patients at this clinic also reported smoking significantly fewer cigarettes per day with significantly fewer self-identifying as smokers and experiencing significantly reduced COPD symptoms.

Conclusion: An interdisciplinary, team-based approach was effective for improving the health of COPD patients in an Appalachian academic primary care practice. ( $\mathrm{J}$ Am Board Fam Med 2020;33:978985.)

Keywords: COPD, Primary Health Care, Quality Improvement, Retrospective Studies, Smokers, West Virginia

\section{Introduction}

Chronic obstructive pulmonary disease (COPD) is the third leading cause of death in the United States. ${ }^{1}$ Age-adjusted mortality has not changed significantly since the 1990s. ${ }^{2}$ Geographically, states clustered along the Ohio and Mississippi Rivers seem to suffer

This article was externally peer reviewed.

Submitted 27 April 2020; revised 25 June 2020; accepted 29 June 2020.

From the Department of Family Medicine, West Virginia University School of Medicine, Robert C. Byrd Health Sciences Center, Morgantown (SP, MMA, AMA, JX, DEK); School of Pharmacy, West Virginia University School of Medicine, Robert C. Byrd Health Sciences Center, Morgantown (MMA, BS)

Funding: None.

Conflict of interest: None.

Corresponding author: Amie M. Ashcraft, PhD, MPH, Department of Family Medicine, West Virginia University School of Medicine, Robert C. Byrd Health Sciences Center, 64 Medical Center Dr, PO, Morgantown, WV 26506 (E-mail: amashcraft@hsc.wvu.edu). disproportionately compared with their national counterparts. $^{2}$ In addition, COPD is estimated to account for over $\$ 49$ billion in direct costs in 2020 with the majority of the cost burden from hospitalization secondary to exacerbations and other concomitant chronic conditions. ${ }^{3}$ Multiple studies predict COPD's prevalence will continue to rise, with 1 estimate predicting an annual increase of $4.8 \%$ per year due to the growing older adult population and the increasing prevalence of tobacco use in various areas globally. ${ }^{4}$

Nationally, West Virginia (WV) ranks among the highest in the nation in poor physical and mental health secondary to limited health care access and services, socioeconomic barriers, and high rates of chronic disease states. ${ }^{5}$ Based on data from the WV Behavioral Risk factor Surveillance System, WV had the highest rates of COPD across the United States (13.9\%). ${ }^{5}$ One of the major drivers to COPD development is tobacco use. Nearly $25 \%$ of 
West Virginians currently smoke, ranking them as the second highest in the nation. ${ }^{5}$ Only about 55\% of these current smokers have previously tried to quit smoking in the past year. ${ }^{5}$ In addition, WV continuously ranks as one of the poorest states in the nation with $\sim 19 \%$ of the population considered to be at or below the poverty line; this economic burden limits the ability for patients to pay and access health care. ${ }^{5}$ This-among other barriers, such as a lack of access to care and cost of COPD inhaler treatment-impacts the success of outpatient COPD management.

Pharmacological treatment regimens should be individualized to the patient, and are often guided by cost, drug availability, ease of use, and patient benefit. It can be difficult for providers to manage a complicated COPD case during a standard 15-to20-minute primary care provider (PCP) office visit - especially in conjunction with other chronic conditions and acute concerns. Many PCPs have transitioned to the "team-based" care approach with the widely accepted implementation of the patient center medical home concept, and it has been demonstrated to improve health, enhance the care experience, and reduce costs in primary care settings. Previous literature has demonstrated the impact of a holistic, team approach for COPD management and beneficial outcomes on patient outcomes. ${ }^{6,7}$ Studies by Koff and colleagues ${ }^{6}$ and Liang and colleagues $^{7}$ demonstrated multidisciplinary care resulted in improved symptoms and quality of life while also decreasing overall health care expenditure compared with standard of care (ie, primary care provider only). This study evaluated the effectiveness of a teambased approach to improving patients' COPD management in a rural primary care clinic in Appalachia.

\section{Methods}

\section{Design}

We conducted a retrospective chart review to assess the impact of our multidisciplinary primary care COPD clinic on selected patient outcomes with approval from the West Virginia University Institutional Review Board. Patients referred to the clinic and having at least 1 visit from February 2015 to February 2019 were included for analysis. Outcome measures include the number of hospitalizations, urgent care visits, and emergency department (ED) utilization (pre- and post-clinic intervention) as well as improvement in symptoms as measured by subjective reporting (as assessed by the COPD Assessment Test [CAT] score, further explanation provided below), rates of smoking cessation, and cigarette use.

\section{Clinic Setting and Procedures}

The Department of Family Medicine (DFM) clinic is located in Morgantown, $\mathrm{WV}$ - a town of approximately 30,000 in Appalachia. The department is a National Committee for Quality Assurance-certified patient-centered medical home and is staffed by 18 physicians, 18 residents, 28 nurses and medical assistants, 1 dietitian and certified tobacco treatment specialist (CTTS), 1 clinical psychologist and psychology intern, 1 licensed clinical social worker, and 2 pharmacists.

The multidisciplinary COPD clinic was implemented within the DFM in early 2015 to reduce COPD admissions and readmissions. The clinic provides consultation with a team composed of a registered dietitian/CTTS, nurse, PharmD, clinical psychologist, family medicine resident, and a Board-certified family medicine attending physician. The multidisciplinary primary care COPD clinic occurs 1 half-day per week (Friday afternoons) in the DFM office space.

Patients are referred to the clinic by their PCP if they meet 1 of the following criteria: 1) newly diagnosed COPD or asthma based on pulmonary function testing; 2) need for tobacco cessation counseling and/or therapy; or 3) recent admission or readmission to the hospital for COPD, asthma, or related pulmonary illness; or 4) medication review. Patients see team members individually during their appointment in the following order: nursing, dietician/ CTTS, psychology, pharmacy, and family medicine. Between each patient/provider interaction, the team regroups for a brief huddle to discuss findings, review treatment options, and manage treatment strategies. Patients' appointments with the full team last approximately 45 to 70 minutes.

At the beginning of each clinic visit, the nurse administers the Fagerström Test for Nicotine Dependence and the COPD Assessment Test (both described below) and informs the patient of the focus of the visit. Then the nurse obtains background information on recent hospitalizations or acute visits, vital signs, medications taken, laboratory results (including pulmonary function tests, computed tomography lung cancer screening, etc.), immunization status, tobacco use (cigarettes, cigars, electronic-cigarettes, snuff, smokeless tobacco, etc.) 
and any other illicit substances, and interest in quitting (as applicable). The dietitian/CTTS then sees the patient to discuss tobacco use and uses motivational interviewing to discuss options for cessation. The CTTS specifically focuses on lifestyle changes to aid with tobacco cessation (eg, increasing water intake, meditation, oral fixation, etc.). She determines and recommends aids to assist with cessation including telephone followup by a team member, referral to the state's 1-800-QUITNOW line, and referral to a free 7-week tobacco cessation class offered at the same clinic. The dietitian/CTTS evaluates unexplained weight loss and recommends supplemental nutrition as indicated, and counsels regarding exercise behaviors to promote exercise tolerance and respiratory capacity. The clinical psychologist then meets with the patient about any prior psychiatric history, barriers to tobacco cessation, and behavioral modification to increase tobacco cessation success. Strategies include but are not limited to schedule modification, journaling, set intentions, etc. The clinical psychologist also reviews barriers for medication adherence if a patient is not taking therapy as prescribed.

The pharmacist reviews the patient's current medication regimen and reconciles as necessary, assesses disease-state burden based on patient-reported symptoms and quality of life, and recommends appropriate changes in therapy. The pharmacist also addresses barriers that could contribute to suboptimal therapy results, including assessing appropriate inhaler administration technique, therapy nonadherence, and medication affordability; recommends appropriate vaccinations; and assists with medication options for tobacco cessation. Then a final huddle with all team members is completed to discuss finalized medication plan, patient barriers, and specific goals. Finally, the resident and attending physicians meet with the patient, confirm history, conduct physical examination, discuss the recommended plan, and arrange for follow-up. Two attending physicians are kept consistent and rotate through the clinic; residents participate as part of their family medicine subspecialty rotation and are variable based on the month.

Followup is determined on an individualized basis. If no changes are made or a patient seems well controlled following hospitalization, they will be referred back to their PCP for future appointments. If further treatment and adjustments are warranted, they will be scheduled for a follow-up appointment within 4 to 6 weeks. The pharmacist or the nurse will contact the patient between appointments, as necessary and based on specific patient needs, to ensure the patient was able to afford and obtain new therapy, tobacco cessation followup, and other clinical issues. As a patient progresses through 2 to 3 follow-up visits to the multidisciplinary primary care COPD clinic, the team will assess whether the patient is ready to be returned back to his PCP for further care or whether consultation with a pulmonary specialist is warranted.

\section{Measures and Data Collection}

Patient data and outcomes were manually collected via chart review of electronic medical record (EMR). Data are included for patients first seen at the COPD specialty clinic between February 1, 2015 and February 28, 2019 to allow assessment of outcomes 6 months after the initial COPD clinic visit. The primary outcomes of the study evaluated frequency of hospitalizations, ED visits, and urgent care visits pre and post enrollment within the COPD specialty clinic. Secondary outcomes evaluated CAT scores and tobacco use pre/post clinic encounters.

\section{Hospital Admissions, ED Visits, and Urgent Care Visits}

Only hospitalizations and urgent care or ED visits which were due to respiratory conditions or complaints-examples include pneumonia, COPD exacerbations, etc.-were included for evaluation. These endpoints were collected via documentation in the EMR in encounter types and externally scanned documents (for hospitalizations which were outside of the system). Patients who also had respiratory complications during hospitalization (ie, not the primary complaint) were also included for analysis, which included hospital-acquired pneumonia or exacerbation following infection. During appointments, patients were asked about any recent hospitalizations, urgent care visits, and ED visits, which was documented at each encounter appointment. Visits and hospitalizations within the health care system were automatically documented within the EMR (during the hospitalization), while those outside of the system had to be reported by the patient or appropriate documentation uploaded by the nursing staff. If a patient had multiple followup appointments with the COPD clinic but a hospitalization occurred between appointments, that 
hospitalization was counted as a postintervention hospitalization.

\section{Fagerström Test for Nicotine Dependence}

At every appointment, patients manually completed a Fagerström Test for Nicotine Dependence. ${ }^{8,9}$ This instrument is standard for assessing the intensity of a patient's physical dependence on nicotine using 6 items that evaluate the number of cigarettes smoked, the compulsion to use nicotine, and nicotine dependence. Patients receive a total score of 0 to 10 , and higher scores indicate more intense physical dependence on nicotine.

\section{COPD Assessment Test}

Symptom improvement was evaluated based on patient-reported surveys, collected as part of standard of care during individual encounters. At each clinic appointment, patients completed a CAT during their rooming to assess the burden of their symptoms and the impact on the patients' daily life. ${ }^{10-14}$ The score is included in the provider's note in the objective section to help monitor disease state management. This questionnaire evaluates cough, phlegm, chest tightness, breathlessness, activity limitations at home, confidence leaving home, sleep, and energy. Patients receive a total score from 0 to 40, with lowers scores indicating fewer symptoms and higher quality of life. Patients who had $\geq 2$ appointments as part of the consult were evaluated based on change in score.

\section{COPD Staging}

COPD staging and classification was based on the 2018 Global Initiative for Chronic Obstructive Lung Disease guidelines. ${ }^{15}$ This classification system evaluates a patient's pulmonary function testing, specifically FEV1, individual CAT score, and recent history of COPD exacerbation or hospitalization. Patients were classified based on stage (1 to 4 ) and grade (A to D).

\section{Smoking Status and Smokeless Tobacco Use}

Patients were classified as smokers and nonsmokers based on their self-reported use of cigarettes. Clinic patients classified as smokers reported their average number of cigarettes/cigars smoked per day. Patients were also classified as smokeless tobacco users (currently used, formerly used, or never used) based on their self-reported use of smokeless tobacco products. End tobacco use status was collected via electronic documentation in the EMR as of September 2019. Tobacco use was assessed during every encounter, as appropriate, and use was updated in the EMR.

\section{Statistical Analysis}

SAS (version 9.4, 2013, SAS Institute, Inc. Cary, $\mathrm{NC)}$ was used to conduct the statistical analyses for this study. Descriptive analyses were performed for demographic and clinical characteristics while paired $t$-test compared the differences in CAT scores and cigarette use between the first and most recent visits. Differences in total hospital admissions, ED visits, and urgent care visits were also evaluated using paired $t$-test. McNemar's test assessed the difference in tobacco status between the first and most recent visits. Tests with a 2 -sided $P<.05$ were considered statistically significant.

\section{Results}

\section{Patient Demographic and Clinical Characteristics}

Since its inception in 2015, a total of 149 patients, ages 38 to 93 years, were seen at the specialty clinic (Table 1). The mean age of patients was $60.2 \pm 10.3$ years. Of the total sample, $83(56 \%)$ were male and $83(56 \%)$ of had private insurance. Patients had on average 1.75 appointments per consult (range, 1 to 6; standard deviation (SD), 1.06). At the first clinic visit, patients' mean Fagerström Test for Nicotine Dependence score was $5.1 \pm 2.3$, and the mean CAT score was $23.3 \pm 8.4$. The majority of patients received a COPD grade of B $(\mathrm{n}=81 ; 62.3 \%)$, followed by $\mathrm{D}(\mathrm{n}=40 ; 30.8 \%), \mathrm{A}$ $(\mathrm{n}=8 ; 6.1 \%)$, and $\mathrm{C}(\mathrm{n}=1 ; 0.8 \%)$. Most patients were classified at a Gold Stage of $2(\mathrm{n}=52 ; 47.3 \%)$. Most patients were tobacco users $(\mathrm{n}=93 ; 62.4 \%)$ and smoked an average of 17.3 cigarettes per day. Finally, the vast majority had never used smokeless tobacco $(n=125$; $86.8 \%)$.

\section{Primary Outcomes: Hospital Admissions, ED Visits, and Urgent Care Visits}

There were significant changes in hospital admissions and ED visits for COPD specialty clinic patients (Table 2). Clinic patients had significantly fewer hospital admissions 6 months after their first clinic visit $(0.09 \pm 0.4)$ than at 6 months before their first visit $(0.3 \pm 0.6),(\mathrm{t}(147)=5.0, P<.0001)$. Clinic patients also had significantly fewer hospital admissions 1 year after their first clinic visit $(0.2 \pm 0.7)$ than at 1 year before their first visit $(0.4 \pm 0.8)$, ( $\mathrm{t}$ $(148)=4.1, P<.0001)$. There was also a significant 
Table 1. Patient Demographic and Clinical Characteristics at First Chronic Obstructive Pulmonary Disease (COPD) Specialty Clinic Visit $(n=149)$

\begin{tabular}{|c|c|}
\hline Patient Characteristics & Mean (SD) \\
\hline Age & $60.2(10.3)$ \\
\hline Cigarettes per day & $17.3(11.2)$ \\
\hline CAT score & $23.3(8.4)$ \\
\hline Nicotine dependence score & $\begin{array}{c}5.1(2.3) \\
\mathrm{n}(\%)\end{array}$ \\
\hline \multicolumn{2}{|l|}{ Sex } \\
\hline Male & $83(56.1)$ \\
\hline Female & $65(43.9)$ \\
\hline \multicolumn{2}{|l|}{ Insurance } \\
\hline Yes & $83(55.7)$ \\
\hline No & $66(44.3)$ \\
\hline \multicolumn{2}{|l|}{ COPD grade } \\
\hline A & $8(6.1)$ \\
\hline B & $81(62.3)$ \\
\hline $\mathrm{C}$ & $1(0.8)$ \\
\hline $\mathrm{D}$ & $40(30.8)$ \\
\hline \multicolumn{2}{|l|}{ Gold stage } \\
\hline 1 & $26(23.6)$ \\
\hline 2 & $52(47.3)$ \\
\hline 3 & $26(23.6)$ \\
\hline 4 & $6(5.5)$ \\
\hline \multicolumn{2}{|l|}{ Smoking } \\
\hline Yes & $93(62.4)$ \\
\hline No & $56(37.6)$ \\
\hline \multicolumn{2}{|l|}{ Smokeless tobacco use } \\
\hline Current user & $5(3.5)$ \\
\hline Former user & $14(9.7)$ \\
\hline Never used & $125(86.8)$ \\
\hline
\end{tabular}

Unequal numbers of patients in some categories due to missing values.

SD, standard deviation; CAT, common admission test.

change in the number of ED visits. Patients at the COPD specialty clinic had significantly fewer ED visits 6 months after their first clinic visit $(0.1 \pm 0.4)$ than 6 months before their first clinic visit $(0.4 \pm 0.7),(\mathrm{t}(148)=5.5, P<.0001)$. Clinic patients had significantly fewer ED visits 1 year after their first clinic visit $(0.2 \pm 0.7)$ than 1 year before $(0.5 \pm 0.9)$, t $(148)=4.3, P<.0001$. There was no difference between pre and post urgent care visits.

\section{Secondary Outcomes: Nicotine Dependence Score, CAT Score, and Tobacco Use Behavior}

There were significant changes in CAT Scores and in the number of cigarettes smoked per day by COPD specialty clinic patients (Table 3). Clinic patients had significantly lower CAT scores at their most recent clinic visit $(20.5 \pm 8.1)$ compared with their first specialty clinic visit $(23.7 \pm 7.5),(\mathrm{t}(55)=3.3$; $P=.002$ ). They also reported smoking significantly fewer cigarettes per day at their most recent clinic visit $(13.8 \pm 11.0)$ compared with their first visit $(17.3 \pm 11.2),(\mathrm{t}(50)=2.3 ; P=.03)$. The percentage of self-identified tobacco users also decreased between their first specialty clinic visit $(61.1 \%)$ to their most recent visit $(52.1 \%), \chi^{2}(1)=11.27 ; P=.0008$.

\section{Discussion}

This study demonstrates the benefit of a multidisciplinary team approach to mitigate complications of COPD and avoid future hospitalizations in a rural primary care setting. Patients referred to the clinic experienced fewer COPD symptoms, smoked less, and had a $50 \%$ reduction in hospitalizations the following year. In light of the huge cost of COPD in the United States annually-an expected \$49 billion in 2020-identifying primary care interventions that can provide prevention of complications is critical. ${ }^{16}$

The current study's findings are consistent with previous literature demonstrating positive benefits from a multidisciplinary approach to care in COPD management. However, only a few trials directly demonstrated reduced hospitalization or

Table 2. Comparisons of the Difference in Total Hospital Admissions, Emergency Department (ED) Visits, and Urgent Care Visits before and after the First Chronic Obstructive Pulmonary Disease (COPD) Specialty Clinic Visit

\begin{tabular}{|c|c|c|c|c|}
\hline & \multicolumn{2}{|c|}{ Mean (SD) } & \multirow[b]{2}{*}{$t(d f)$} & \multirow[b]{2}{*}{$P$-Value* } \\
\hline & Before & After & & \\
\hline \multicolumn{5}{|l|}{6 month } \\
\hline $\begin{array}{l}\text { Hospital } \\
\text { admissions }\end{array}$ & $0.3(0.6)$ & $0.09(0.4)$ & $5.0(147)$ & $<.0001^{\ddagger}$ \\
\hline ED visits & $0.4(0.7)$ & $0.1(0.4)$ & $5.5(148)$ & $<.0001^{\ddagger}$ \\
\hline $\begin{array}{l}\text { Urgent care } \\
\text { visits } †\end{array}$ & - & - & - & - \\
\hline \multicolumn{5}{|l|}{1 year } \\
\hline $\begin{array}{l}\text { Hospital } \\
\text { admissions }\end{array}$ & $0.4(0.8)$ & $0.2(0.7)$ & $4.1(148)$ & $<.0001^{\ddagger}$ \\
\hline ED visits & $0.5(0.9)$ & $0.2(0.7)$ & $4.3(148)$ & $<.0001^{\ddagger}$ \\
\hline $\begin{array}{l}\text { Urgent care } \\
\text { visits }\end{array}$ & $0.05(0.3)$ & 0 & $1.8(148)$ & .07 \\
\hline
\end{tabular}

${ }^{*} P$-value from paired sample $t$-test, $\ddagger P<.001$.

${ }^{\dagger}$ There were not enough available data to compare urgent care visits six months before and after the first specialty clinic visit. df, degrees of freedom. 
Table 3. Comparison of Mean Difference Nicotine Dependency Scores, Common Admission Test (CAT) Scores, and Cigarette Use/Day before and after the Most Recent Chronic Obstructive Pulmonary Disease (COPD) Clinic Visit

\begin{tabular}{lllll}
\hline & \multicolumn{2}{c}{ Mean (SD) } & & \\
\cline { 2 - 3 } & Before & After & $\mathrm{t}(\mathrm{df})$ & $P$-Value \\
\hline $\begin{array}{l}\text { Nicotine } \\
\text { dependency } \\
\text { scores }\end{array}$ & $4.7(2.1)$ & $4.2(2.1)$ & $1.0(22)$ & 0.32 \\
$\begin{array}{l}\text { CAT scores } \\
\text { Cigarette use/day }\end{array}$ & $23.7(7.5)$ & $20.5(8.1)$ & $3.3(55)$ & $0.002 \neq$ \\
\hline
\end{tabular}

${ }^{*} P$-value from paired sample $t$-test, $\dagger P<.05, \ddagger P<.01$. $\mathrm{SD}$, standard deviation; df, degrees of freedom.

rehospitalization; most have concentrated on other outcome measurements including quality of life and symptom improvement. ${ }^{6,7,17-19}$ The recent evidence of the integrated care management approach further supports the benefit of a multidisciplinary team to reduce $\mathrm{ED}$ visits and hospital admissions. ${ }^{20}$ The streamlined unified meaningfully managed interdisciplinary team (SUMMIT) trial protocol shows further interest in the multidisciplinary approach to care in an urban homelessness setting; results of the trial are not yet available. ${ }^{21}$ In contrast, the current study demonstrated reduced COPD hospitalization rates in a primary care rural clinic setting.

The current study also demonstrated a significant improvement in CAT scores as well as increased tobacco cessation rates and decreased cigarette use. Success rates for tobacco cessation in this study may be due to the multiple encounters reinforcing the cessation message including counseling by the certified cessation specialist, the pharmacist, the psychologist, and the physicians. Patients may have also adjusted behaviors exclusively during this limited, intensive intervention, knowing they were being proactively monitored and observed (Hawthorne effect); future studies could assess long-term impact of an interdisciplinary care on outcomes such as sustained tobacco cessation. In addition, some of these outcomes, for example, those included in Table 3, have few data points, and therefore the results should be interpreted with caution.

Members of the multidisciplinary team are not always consistent in the studies cited. Most contained at least a physician, nursing and/or care manager, and respiratory/tobacco cessation specialist. The inclusion of a physical therapist, pharmacist, psychologist, dietitian, exercise specialists, and social workers varied by

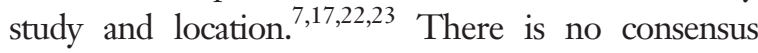
agreement regarding the ideal makeup of a COPD team despite the multiple studies that have been conducted. Regardless of the disciplines involved, teams should establish interdependence among the members where the treatment plan and patient-specific goals combines the expertise of the individual disciplines involved. The current study site has 6 core disciplines consistently represented-medicine, pharmacy, dietitian, tobacco cessation, psychology, and nursing - a combination that has worked well for our workflow and the site's currently available space. For practice sites interested in starting a multidisciplinary clinic, targeted strategies to identify disciplinary buy-in, evaluate gaps in care, develop an implementation plan, and discuss a clinic workflow are critical first steps for success. ${ }^{24,25}$

COPD's morbidity and mortality have significant implications in the rural setting. Based on a report from the Centers for Disease Control and Prevention, death due to respiratory illness is significantly higher in rural populations compared with urban settings. ${ }^{26}$ The report hypothesizes this trend is due to health care access barriers including preventative and screening services, treatment delays, and lack of emergency services. Implementing strategies to bridge this gap are needed. Utilization of intensive interdisciplinary clinics may provide benefit and broadened scope in areas where physician resources may be limited.

This study has several limitations including the length of followup. The clinic has been in operation since 2015, so sustained improvement cannot be ascertained longer term. In addition, most patients had 3 visits to the COPD clinic and were then returned to the care of their PCP; outcomes regarding those referred for specialty care were too few for analysis. In addition, the study data were limited to chart review and patient reporting. Hospitalization rates were accurately documented if within our health care system; however, if a patient was admitted to an outside facility, documentation was based on patient-reports or if the institution notified our clinic. This was also viewed with the Fagerström score reporting; despite this being incorporated in the procedure of rooming a patient, it was inconsistently documented in the EMR. As a result, some data were missing, and we evaluated the difference from first visit to most recent encounter instead of changes between visits. We also 
recognize the limitations of using the CAT score to measure symptom improvement-primarily the subjectivity of the rating score and difficulty some patients experience in ranking symptoms on a Likert scale (ie, what constitutes a ranking of 2 vs 3 for coughing). However, the CAT score is a validated tool for assessment of COPD symptoms and included in the guidelines as recommended assessment for staging and monitoring. ${ }^{15}$ Finally, tobacco cessation and cigarette use was also self reported and not verified via carbon monoxide or other measurement. This may be a consideration in future evaluation to verify cessation rates.

\section{Conclusions}

The implications of these findings may improve important COPD-related outcomes including readmission hospitalizations. While causality cannot be established in this study, the results are promising and further investigation is warranted. The teambased model should be considered in other practice settings to help reduce admissions and disease management. Further research is needed to evaluate the long-term implementation of the team-approach, establish an objective method to measure patient symptoms, and determine an effective strategy to spread the approach throughout primary care.

The authors would sincerely like to thank the faculty, residents, nurses, clinic staff, and students for their hard work and dedication to this effort; in particular, Kara Kozul, Kelsey Samek, and Judy Siebart.

To see this article online, please go to: http://jabfm.org/content/ 33/6/978.full.

\section{References}

1. Centers for Disease Control and Prevention. Deaths: final data for 2017. National vital statistics reports. 2019. Available from: https://www.cdc.gov/ nchs/data/nvsr/nvsr68/nvsr68_09_tables-508.pdf.

2. Centers for Disease Control and Prevention. COPD death rates in the United States. 2017. Available from: https://www.cdc.gov/copd/data.html.

3. Ford ES, Murphy LB, Khavjou O, Giles WH, Holt JB, Croft JB. Total and state-specific medical and absenteeism costs of COPD among adults aged $\geq$ 18 years in the United States for 2010 and projections through 2020. Chest 2015;147:31-45.

4. Khakban A, Sin DD, FitzGerald JM, et al. The projected epidemic of chronic obstructive pulmonary disease hospitalizations over the next 15 years: a population health perspective. Am J Respir Crit Care Med 2017;195:287-91.
5. WV Department of Health and Human Resources. Fast Facts from WV BRFSS. 2019. Available from: https:// dhhr.wv.gov/hpcd/data_reports/Pages/Fast-Facts.aspx.

6. Koff PB, Jones RH, Cashman JM, Voelkel NF, Vandivier RW. Proactive integrated care improves quality of life in patients with COPD. Eur Respir J 2009;33:1031-8.

7. Liang J, Abramson MJ, Russell G, et al. Interdisciplinary COPD intervention in primary care: a cluster randomized controlled trial. Eur Respir J 2019;53:1801530.

8. Pomerleau CS, Majchrzak MJ, Pomerleau OF. Nicotine dependence and the Fagerström Tolerance Questionnaire: a brief review. J Subst Abuse 1989;1:471-7.

9. Heatherton TF, Kozlowski LT, Frecker RC, Fagerström KO. The Fagerström Test for Nicotine Dependence: a revision of the Fagerström Tolerance Questionnaire. Br J Addict 1991;86:1119-27.

10. Jones P, Harding G, Wiklund I, Berry P, Leidy N. Improving the process and outcome of care in COPD: development of a standardised assessment tool. Prim Care Respir J 2009;18:208-15.

11. Jones PW, Harding G, Berry P, Wiklund I, Chen W-H, Kline Leidy N. Development and first validation of the COPD Assessment Test. Eur Respir J 2009;34:648-54.

12. Jones PW, Tabberer M, Chen W. Creating scenarios of the impact of COPD and their relationship to COPD assessment test (CATTM) scores. BMC Pulm Med 2011;11:42.

13. Jones PW, Harding G, Wiklund I, et al. Tests of the responsiveness of the chronic obstructive pulmonary disease (COPD) assessment Test (CAT) following acute exacerbations and pulmonary rehabilitation. Chest 2012;142:134-40.

14. Gupta N, Pinto LM, Morogan A, Bourbeau J. The COPD assessment test: a systematic review. Eur Respir J 2014;44:873-84.

15. Global Initiative for Chronic Obstructive Lung Disease (GOLD). Global strategy for the diagnosis, management, and prevention of chronic obstructive pulmonary disease: 2020 Report. 2020. Available from: https://goldcopd.org/wp-content/uploads/2019/ 12/GOLD-2020-FINAL-ver1.2-03Dec19_WMV.pdf.

16. Joszt L. Drivers of high hospital costs for patients with COPD. 2017. Available from: https://www. ajmc.com/newsroom/drivers-of-high-hospital-costsfor-patients-with-copd.

17. Casas A, Troosters T, Garcia-Aymerich J, et al. Integrated care prevents hospitalisations for exacerbations in COPD patients. Eur Respir J 2006;28:123-30.

18. Wynn N, Johnson N, Ferreira TBD. Multidisciplinary team approach to reduce COPD readmissions in a tertiary care center. AmJ Resp Crit Care Med 2018;197:A4977.

19. Dajczman E, Robitaille C, Ernst $P$, et al. Integrated interdisciplinary care for patients with chronic obstructive pulmonary disease reduces emergency 
department visits and admissions: a quality assurance study. Can Resp J 2013;20:351-6.

20. Bandurska E, Damps-Konstanska I, Popowski P, et al. Cost-effectiveness analysis of integrated care in management of advanced chronic obstructive pulmonary disease (COPD). Med Sci Monit 2019;25:2879-85.

21. Chan B, Edwards ST, Devoe M. The SUMMIT ambulatory-ICU primary care model for medically and socially complex patients in an urban federally qualified health center: study design and rationale. Addict Sci Clin Pract 2018;13:27.

22. Braman SS, Lee DW. Primary care management of chronic obstructive pulmonary disease: an integrated goal-directed approach. Curr Opin Pulm Med 2010;16:83-8.
23. Kuzma AM, Meli Y, Meldrum C, Jellen P, et al. Multidisciplinary care of the patient with chronic obstructive pulmonary disease. Proc Am Thorac Soc 2008;5:567-71.

24. Mitchell GK, Tieman JJ, Shelby-James TM. Multidisciplinary care planning and teamwork in primary care. Med J Aust 2008;188:S61-S64.

25. Schottenfeld L, Petersen D, Peikes D, et al. Creating patient-centered team-based primary care. Princeton, NJ: Mathematica Policy Research. AHRQ Publication No. 16-0002-EF. 2016.

26. Garcia MC, Faul M, Massetti G, et al. Reducing potentially excess deaths from the five leading causes of death in the rural United States. MMWR Surveill Summ 2017;66:1-7. 